\title{
Increased Permeability-Glycoprotein Inhibition at the Human Blood-Brain Barrier Can Be Safely Achieved by Performing PET During Peak Plasma Concentrations of Tariquidar
}

\author{
William C. Kreisl ${ }^{1,2}$, Ritwik Bhatia ${ }^{1}$, Cheryl L. Morse ${ }^{1}$, Alicia E. Woock ${ }^{1}$, Sami S. Zoghbi ${ }^{1}$, H. Umesha Shetty ${ }^{1}$, \\ Victor W. Pike ${ }^{1}$, and Robert B. Innis ${ }^{1}$ \\ ${ }^{I}$ Molecular Imaging Branch, National Institute of Mental Health, Bethesda, Maryland; and ${ }^{2}$ Taub Institute, Columbia University \\ Medical Center, New York, New York
}

The permeability-glycoprotein (P-gp) efflux transporter is densely expressed at the blood-brain barrier, and its resultant spare capacity requires substantial blockade to increase the uptake of avid substrates, blunting the ability of investigators to measure clinically meaningful alterations in P-gp function. This study, conducted in humans, examined 2 P-gp inhibitors (tariquidar, a known inhibitor, and disulfiram, a putative inhibitor) and 2 routes of administration (intravenous and oral) to maximally increase brain uptake of the avid and selective $\mathrm{P}$-gp substrate ${ }^{11} \mathrm{C}-\mathrm{N}$-desmethyl-loperamide (dLop) while avoiding side effects associated with high doses of tariquidar. Methods: Forty-two ${ }^{11} \mathrm{C}$-dLop PET scans were obtained from 37 healthy volunteers. PET was performed with ${ }^{11} \mathrm{C}$-dLop under the following 5 conditions: injected under baseline conditions without P-gp inhibition, injected $1 \mathrm{~h}$ after intravenous tariquidar infusion, injected during intravenous tariquidar infusion, injected after oral tariquidar, and injected after disulfiram. ${ }^{11} \mathrm{C}$-dLop uptake was quantified with kinetic modeling using metabolite-corrected arterial input function or by measuring the area under the time-activity curve in the brain from 10 to 30 min. Results: Neither oral tariquidar nor oral disulfiram increased brain uptake of ${ }^{11} \mathrm{C}$-dLop. Injecting ${ }^{11} \mathrm{C}$-dLop during tariquidar infusion, when plasma tariquidar concentrations reach their peak, resulted in a brain uptake of the radioligand approximately 5 -fold greater than baseline. Brain uptake was similar with 2 and $4 \mathrm{mg}$ of intravenous tariquidar per kilogram; however, the lower dose was better tolerated. Injecting ${ }^{11} \mathrm{C}$-dLop after tariquidar infusion also increased brain uptake, though higher doses (up to $6 \mathrm{mg} / \mathrm{kg}$ ) were required. Brain uptake of ${ }^{11} \mathrm{C}$-dLop increased fairly linearly with increasing plasma tariquidar concentrations, but we are uncertain whether maximal uptake was achieved. Conclusion: We sought to increase the dynamic range of P-gp function measured after blockade. Performing ${ }^{11} \mathrm{C}$-dLop PET during peak plasma concentrations of tariquidar, achieved with concurrent administration of intravenous tariquidar, resulted in greater P-gp inhibition at the human blood-brain barrier than delayed administration and allowed the use of a lower, more tolerable dose of tariquidar. On the basis of prior monkey studies, we suspect that plasma concentrations of tariquidar did not fully block P-gp; however, higher doses of tariquidar would likely be associated with unacceptable side effects.

Received Aug. 6, 2014; revision accepted Nov. 13, 2014.

For correspondence or reprints contact: William C. Kreisl, Taub Institute, Columbia University Medical Center, 622 W. 168th St., PH 19th Floor, New York, NY 10032.

E-mail: wck2107@cumc.columbia.edu

Published online Dec. 11, 2014.

COPYRIGHT (c) 2015 by the Society of Nuclear Medicine and Molecular Imaging, Inc.
Key Words: P-glycoprotein (P-gp); positron emission tomography (PET); N-desmethyl-loperamide; tariquidar

J Nucl Med 2015; 56:82-87

DOI: 10.2967/jnumed.114.146894

$\mathbf{T}$ he efflux transporter P-glycoprotein (P-gp) is a component of the blood-brain barrier (BBB) and a proposed mechanism of drug resistance (1). Under physiologic conditions, P-gp acts at high capacity, completely blocking entry into the brain of avid substrates, including radiolabeled substrates. More than $50 \%$ of P-gp transporters may need to be inhibited pharmacologically to increase uptake of radiolabeled substrates into a range measurable by PET (2). Notably, the P-gp inhibitor tariquidar has been shown to increase brain uptake of the substrate PET radiotracers ${ }^{11} \mathrm{C}$ - $N$-desmethyl-loperamide $\left({ }^{11} \mathrm{C}\right.$-dLop) and ${ }^{11} \mathrm{C}$ - $(R)$-verapamil 2- to 3-fold, compared with baseline (3-6).

To detect pathologic overexpression of P-gp using PET, normal P-gp function (which is already of high capacity) must be distinguished from P-gp overexpression (which has even higher capacity). Treating subjects with a fixed dose of tariquidar before PET imaging would hypothetically increase radiotracer uptake into a dynamic range at which differential response to inhibition-caused by differential P-gp function - could be measured.

To achieve increases in radiotracer brain uptake into this range, however, past studies have required high intravenous doses $(>2 \mathrm{mg} / \mathrm{kg}$ ) of tariquidar that were associated with adverse drug reactions $(4,7,8)$. In addition, because these studies administered the PET radiotracer after completion of tariquidar infusion, they may have missed the window of peak P-gp inhibition and, thus, the most reliable measurement of increased P-gp inhibition. Although tariquidar is the only third-generation P-gp inhibitor that has been found to be safe for human administration, other potential methods of pharmacologic inhibition of P-gp include oral administration of tariquidar (9) or oral administration of disulfiram, a drug whose metabolites have been shown to inhibit P-gp in vitro (10-12).

This study sought to determine whether increased inhibition of P-gp at the human BBB could be safely achieved and reliably measured by changing the timing or route of administration of tariquidar or using disulfiram. ${ }^{11} \mathrm{C}$-dLop PET was performed on healthy volunteers under baseline conditions or after P-gp inhibition. For the P-gp blocked condition, ${ }^{11} \mathrm{C}$-dLop was injected $1 \mathrm{~h}$ 
after intravenous tariquidar infusion, during intravenous tariquidar infusion, after oral tariquidar, or after disulfiram.

\section{MATERIALS AND METHODS}

Thirty-seven healthy volunteers (mean age \pm SD, $29.1 \pm 6.9$ y; 18 women, 19 men) were included. Volunteers with serious medical or psychiatric illness, those using illicit drugs, and those taking medications other than oral contraceptives were excluded. The study was approved by the National Institutes of Health Combined Neurosciences Institutional Review Board. All participants gave written informed consent before entering the study. Subjects were monitored for changes in blood pressure, temperature, heart rate, and respiration rate before and after tariquidar infusion. These parameters, as well as electrocardiogram tracing, were similarly monitored after ${ }^{11} \mathrm{C}$-dLop injection. Blood and urine laboratory tests were repeated within $24 \mathrm{~h}$ of study completion. Plasma was drawn to measure total tariquidar concentration for 16 subjects and free fraction of tariquidar for 5 subjects. Methods for measuring total and free concentration of tariquidar are given in the supplemental materials (supplemental materials are available at http:// jnm.snmjournals.org).

\section{${ }^{11}$ C-dLop Imaging}

${ }^{11} \mathrm{C}$-dLop was prepared by the methylation of its primary amide precursor with ${ }^{11} \mathrm{C}$-iodomethane, as described previously (13). The radiotracer was obtained in high radiochemical purity $(100 \%)$ and with a specific activity of $101 \pm 50 \mathrm{GBq} / \mu \mathrm{mol}$ at the time of injection.

Fourteen ${ }^{11} \mathrm{C}$-dLop scans were obtained without P-gp inhibition (baseline).The remaining 28 scans were obtained with P-gp inhibition. Methods for PET imaging and tariquidar preparation are given in supplemental materials.

Intravenous Tariquidar with Delayed Radiotracer Injection. Scan data from 7 subjects who received either $4 \mathrm{mg} / \mathrm{kg}$ or $6 \mathrm{mg} / \mathrm{kg}$ of intravenous tariquidar in a previously published study (4) were included in this study for comparison. For these subjects, ${ }^{11} \mathrm{C}$-dLop was injected approximately $60 \mathrm{~min}$ after completion of the tariquidar infusion.

Intravenous Tariquidar with Concurrent Radiotracer Injection. Twelve subjects received intravenous tariquidar at doses of either $2 \mathrm{mg} / \mathrm{kg}$ ( $n=10$; 4 women; body weight, $71.2 \pm 18.7 \mathrm{~kg}$ ) or $4 \mathrm{mg} / \mathrm{kg}$ ( $n=2$; 1 woman; body weight, $72.8 \pm 19.4 \mathrm{~kg}$ ). Subjects had 2 intravenous catheters placed (1 in each antecubital vein) before imaging. Under both dose conditions, tariquidar was infused at a rate of $4 \mathrm{mg} / \mathrm{kg} / \mathrm{h}$ through 1 catheter. Halfway through the tariquidar infusion (at $30 \mathrm{~min}$ for the $4 \mathrm{mg} / \mathrm{kg}$ group and at $15 \mathrm{~min}$ for the $2 \mathrm{mg} / \mathrm{kg}$ group), ${ }^{11} \mathrm{C}$-dLop was injected through the other catheter. Arterial sampling was not performed.

Oral Tariquidar Administration. To determine the dose strategy for oral tariquidar, we performed pharmacokinetic studies in healthy volunteers (supplemental materials). On the basis of the results, 3 additional subjects (all men; body weight, $76.1 \pm 16.5 \mathrm{~kg}$ ) received oral tariquidar at a dose of $1,500 \mathrm{mg} 10-12 \mathrm{~h}$ before ${ }^{11} \mathrm{C}$-dLop injection. Arterial sampling was not performed.

Oral Disulfiram Administration. Disulfiram was administered at 2 doses: $500 \mathrm{mg}$ of oral disulfiram as a 1-time dose $10 \mathrm{~h}$ before ${ }^{11} \mathrm{C}$-dLop injection ( $n=3$; all men; body weight, $83.4 \pm 12.0 \mathrm{~kg}$ ) or $2.5 \mathrm{~g}$ over $4 \mathrm{~d}$ before ${ }^{11} \mathrm{C}$-dLop injection $(n=3$; all women; body weight, $78.1 \pm$ $24.6 \mathrm{~kg}$ ). Subjects were given written and oral instructions to abstain from alcohol-containing products for $2 \mathrm{~d}$ prior to and $2 \mathrm{wk}$ after disulfiram administration. Arterial sampling was performed.

\section{${ }^{11} \mathrm{C}$-dLop Image Analysis}

Reconstructed PET images were realigned and normalized to stereotactic space using Pixelwise Modeling Software 3.0 (PMOD Technologies Ltd.). The Montreal Neurologic Institute template was used to define a region of composite neocortex by creating a weighted average of activity from the frontal, parietal, occipital, and temporal cortices. Regions were manually adjusted when necessary to exclude cerebrospinal fluid space and choroid plexus.

The concentration of radioactivity was expressed as standardized uptake value $(\mathrm{SUV})=$ (measured activity per $\mathrm{cm}^{3}$ brain/injected activity $) \cdot(\mathrm{g}$ of body weight). The area under the time-activity curve from 10 to $30 \mathrm{~min}\left(\mathrm{AUC}_{10-30}\right)$ for each composite brain region was calculated using the trapezoidal method.

For those subjects with metabolite-corrected plasma data, rate constants $\left(K_{1}\right.$ and $\left.k_{2}\right)$ and total distribution volume $\left(V_{\mathrm{T}}\right)$ were calculated using a 1-tissue-compartmental model. The total concentration of radioactivity in whole blood was used for vascular correction, assuming that blood constitutes $5 \%$ brain volume.

Because the posterior pituitary lacks a BBB and prior baseline PET scans have shown high uptake of ${ }^{11} \mathrm{C}$-dLop in this organ (6), we measured activity in the pituitary gland to estimate brain uptake of ${ }^{11} \mathrm{C}$ dLop in complete absence of P-gp (supplemental materials).

\section{Statistical Analysis}

Statistical analysis was performed using SPSS 17.0 (IBM). Group differences in ${ }^{11} \mathrm{C}$-dLop uptake were evaluated using the Mann-Whitney $U$ test. To determine whether the tariquidar $-{ }^{11} \mathrm{C}$-dLop dose-response curve best fit a linear or nonlinear model, we used the Curve Estimation function in SPSS to perform linear and nonlinear (quadratic, cubic, and sigmoidal) regressions. The estimated half maximum effective concentration $\left(\mathrm{EC}_{50}\right)$ value and its $95 \%$ confidence interval were determined with the R software package (www.r-project.org) using a lognormal dose-response model.

\section{RESULTS}

\section{Safety Analysis}

Tariquidar was generally well tolerated in subjects who received the lowest doses ( $2 \mathrm{mg} / \mathrm{kg}$ intravenously and $1,500 \mathrm{mg}$ orally). Two subjects who received $2 \mathrm{mg} / \mathrm{kg}$ intravenously reported a mild metallic taste during the tariquidar infusion. Two subjects who received $4 \mathrm{mg}$ of intravenous tariquidar per kilogram experienced light-headedness and conjunctival injection, and 1 of these subjects had a brief syncopal episode immediately after the PET scan that resolved without sequelae. One subject who received $6 \mathrm{mg}$ of intravenous tariquidar per kilogram complained of a metallic taste accompanied by nausea that resolved once tariquidar infusion was stopped.

Disulfiram was well tolerated by all subjects. One subject experienced a mild, transient disulfiram-ethanol reaction $3 \mathrm{~d}$ after the PET scan due to unwittingly consuming food prepared with wine.

\section{Imaging Results}

When ${ }^{11} \mathrm{C}$-dLop was injected in the baseline condition, uptake of activity in the brain was negligible, in agreement with previous studies. Brain time-activity curves reached a plateau at less than $0.2 \mathrm{SUV}$ (Fig. 1). Both $K_{1}$ and $k_{2}$ values were low, resulting in $V_{\mathrm{T}}$ values of $1.20 \pm 0.16$. $\mathrm{AUC}_{10-30}$ values for composite neocortex were $2.94 \pm 0.66 \mathrm{SUV} \cdot \mathrm{min}$ (Table 1). Individual region-ofinterest values are shown in Supplemental Table 1.

When ${ }^{11} \mathrm{C}$-dLop was injected approximately $60 \mathrm{~min}$ after tariquidar infusion, brain uptake of ${ }^{11} \mathrm{C}$-dLop was approximately 2to 4-fold greater than at baseline (Table 1). Brain time-activity curves reached plateaus of approximately 0.3 and approximately 0.6 SUV during delayed administration of tariquidar at 4 and $6 \mathrm{mg} / \mathrm{kg}$ intravenously, respectively. The resulting $\mathrm{AUC}_{10-30}$ values for composite neocortex were 1.7-3.4 times greater than baseline.

When ${ }^{11} \mathrm{C}$-dLop was injected during infusion of tariquidar, brain uptake of ${ }^{11} \mathrm{C}$-dLop was approximately 5 -fold greater than 


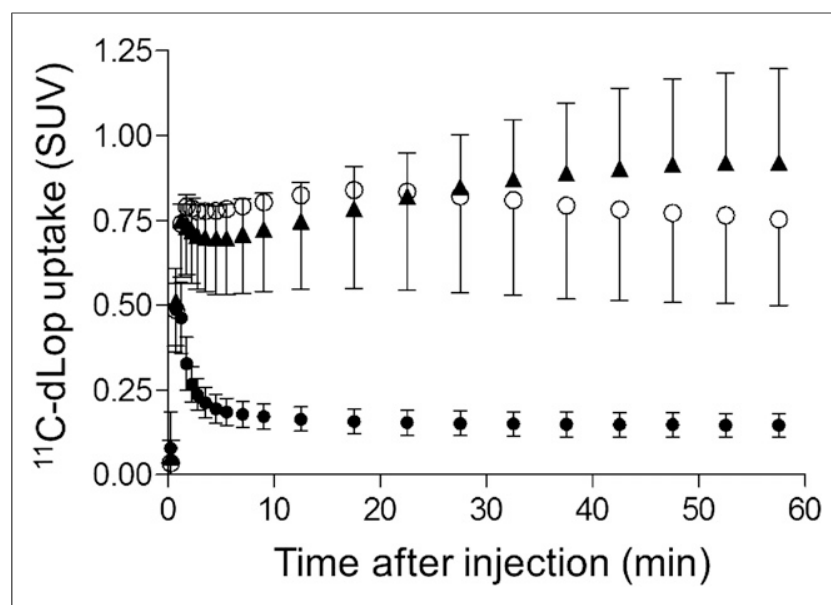

FIGURE 1. Composite neocortex time-activity curves showing brain uptake of radioactivity after ${ }^{11} \mathrm{C}$-dLop injection. ${ }^{11} \mathrm{C}$-dLop was injected at baseline $(\bullet)$ and during intravenous infusion of tariquidar at $2(\bigcirc)$ and $4 \mathrm{mg} / \mathrm{kg}(\boldsymbol{\Lambda})$. Error bars denote SD.

at baseline (Figs. 1 and 2; Table 1). Brain time-activity curves reached plateaus of $0.4-1.4$ and $0.7-1.1$ SUV during concurrent administration of tariquidar at 2 and $4 \mathrm{mg} / \mathrm{kg}$ intravenously, respectively. The resulting $\mathrm{AUC}_{10-30}$ values for composite neocortex were approximately 5 times greater than baseline, approximately 3 times greater than delayed administration of $4 \mathrm{mg}$ of intravenous tariquidar per kilogram, and approximately 1.5 times greater than delayed administration of $6 \mathrm{mg}$ of intravenous tariquidar per kilogram. At baseline, ${ }^{11} \mathrm{C}$-dLop uptake in the pituitary was 17 times greater than in the composite neocortex (Supplemental Fig. 1). After P-gp inhibition, uptake in the pituitary was 3.5 times greater than in composite neocortex. ${ }^{11} \mathrm{C}$-dLop uptake in the pituitary was similar at baseline and after P-gp inhibition $(\sim 2.7$ vs. $\sim 2.5$ SUV $)$.

Oral tariquidar did not increase brain uptake of ${ }^{11} \mathrm{C}$-dLop. Measured values for $\mathrm{AUC}_{10-30}$ did not differ from those in baseline scans (Fig. 3; Table 1). Results from the pharmacokinetic study for oral tariquidar are given in the supplemental materials.
Neither low- nor high-dose disulfiram administration was associated with ${ }^{11} \mathrm{C}$-dLop uptake into brain greater than baseline (Fig. 4; Table 1). Disulfiram did not result in changes in $V_{\mathrm{T}}, K_{1}, k_{2}$, or $\mathrm{AUC}_{10-30}$. The plasma-free fraction $\left(f_{\mathrm{p}}\right)$ of ${ }^{11} \mathrm{C}$-dLop was slightly lower in disulfiram-treated subjects than in subjects who had ${ }^{11} \mathrm{C}$-dLop under baseline conditions $(12 \% \pm 1 \%$ vs. $15 \% \pm 2 \% ; P=0.0426$, MannWhitney $U$ test). However, $V_{\mathrm{T}} / f_{\mathrm{p}}$ values did not differ between disulfiram-treated and baseline subjects $(P=0.2284)$.

\section{Dose-Response of Tariquidar on ${ }^{11} \mathrm{C}$-dLop Uptake}

We measured the plasma concentrations of tariquidar for subjects who had concurrent radiotracer injection, oral tariquidar, and 1 subject who had delayed radiotracer injection. For the concurrent group, plasma concentrations of tariquidar were $1,040 \pm 360 \mathrm{ng} / \mathrm{mL}$ $(1,609 \pm 556 \mathrm{nM})$ for the $2 \mathrm{mg} / \mathrm{kg}$ dose and $1,438 \pm 528 \mathrm{ng} / \mathrm{mL}$ $(2,224 \pm 817 \mathrm{nM})$ for the $4 \mathrm{mg} / \mathrm{kg}$ dose. Plasma concentrations of tariquidar after oral administration were $133 \pm 46 \mathrm{ng} / \mathrm{mL}(205 \pm$ $71 \mathrm{nM}$ ), or only $13 \%$ of the concentration achieved with concurrent administration of $2 \mathrm{mg} / \mathrm{kg}$ intravenously. The subject who had intravenous tariquidar $(4 \mathrm{mg} / \mathrm{kg})$ with delayed radiotracer injection had plasma concentrations of $232 \mathrm{ng} / \mathrm{mL}(358 \mathrm{nM})$, or $22 \%$ of the mean value for subjects who had concurrent injection of radiotracer at the same dose of tariquidar. The free fraction of tariquidar was $0.0054 \pm 0.0002$ for the 5 subjects who had ${ }^{11} \mathrm{C}$-dLop imaging and $0.0053 \pm 0.0003$ for all 21 subjects who had free fraction measured.

${ }^{11} \mathrm{C}$-dLop uptake into the brain increased linearly, with the total concentration of tariquidar at low and moderate concentrations of the inhibitor; however, at higher concentrations, the PET response appeared to plateau (Fig. 5). Goodness of fit was greater for the sigmoidal model $\left(r^{2}=0.81\right)$ than linear $\left(r^{2}=0.30\right)$, quadratic $\left(r^{2}=0.65\right)$, or cubic $\left(r^{2}=0.66\right)$ models. The estimated $\mathrm{EC}_{50}$ value and its $95 \%$ confidence interval were $306 \mathrm{ng} / \mathrm{mL}$ and 20 592, respectively. The estimated maximum for ${ }^{11} \mathrm{C}$-dLop uptake and its $95 \%$ confidence interval were $15 \mathrm{SUV} \cdot \mathrm{min}$ and 12-19, respectively. This maximum corresponds to peak uptake of 0.8 SUV in the brain. Brain uptake of the radiotracer did not correlate with the free concentration of tariquidar in plasma $(r=-0.61$, $P=0.28$ ); however, free fraction was measured in only 5 subjects.

TABLE 1

Uptake of ${ }^{11} \mathrm{C}$-dLop in Composite Neocortex at Baseline and After Pharmacologic Inhibition of P-Glycoprotein

\begin{tabular}{|c|c|c|c|c|}
\hline Condition & $\mathrm{AUC}_{10-30}(\mathrm{SUV} \cdot \mathrm{min})$ & $K_{1}\left(\mathrm{~mL} \cdot \mathrm{cm}^{-3} \cdot \mathrm{min}^{-1}\right)$ & $k_{2}\left(\min ^{-1}\right)$ & $V_{\mathrm{T}}\left(\mathrm{mL} \cdot \mathrm{cm}^{-3}\right)$ \\
\hline Baseline $(n=14)$ & $2.94 \pm 0.66$ & $0.014 \pm 0.002$ & $0.012 \pm 0.002$ & $1.20 \pm 0.16$ \\
\hline \multicolumn{5}{|l|}{ Tariquidar } \\
\hline $4 \mathrm{mg} / \mathrm{kg}$ concurrent $(n=2)$ & $14.61 \pm 2.31$ & & & \\
\hline $2 \mathrm{mg} / \mathrm{kg}$ concurrent $(n=10)$ & $15.34 \pm 5.25^{\star}$ & & & \\
\hline $1,500 \mathrm{mg}$ oral $(n=3)$ & $2.06 \pm 0.07$ & & & \\
\hline $4 \mathrm{mg} / \mathrm{kg}$ delayed $(n=4)$ & $5.26 \pm 2.26^{\dagger}$ & $0.026 \pm 0.009$ & $0.010 \pm 0.001$ & $2.56 \pm 0.64$ \\
\hline $6 \mathrm{mg} / \mathrm{kg}$ delayed $(n=3)$ & $10.05 \pm 3.25^{\ddagger}$ & $0.049 \pm 0.011$ & $0.013 \pm 0.001$ & $3.88 \pm 0.71$ \\
\hline \multicolumn{5}{|l|}{ Disulfiram } \\
\hline $500 \mathrm{mg}(n=3)$ & $3.13 \pm 0.43$ & $0.012 \pm 0.001$ & $0.011 \pm 0.001$ & $1.14 \pm 0.03$ \\
\hline $2,500 \mathrm{mg}(n=3)$ & $2.44 \pm 0.12$ & $0.012 \pm 0.007$ & $0.008 \pm 0.003$ & $1.53 \pm 0.66$ \\
\hline \multicolumn{5}{|c|}{$\begin{array}{l}{ }^{\star} P<0.0001 \text { vs. baseline. } \\
{ }^{\dagger} P=0.06 \text { vs. baseline. } \\
{ }^{\ddagger} P=0.003 \text { vs. baseline. } \\
K_{1}=\text { rate constant for brain entry; } k_{2}=\text { rate constant for brain efflux. } \\
\text { Data are mean } \pm S D .\end{array}$} \\
\hline
\end{tabular}




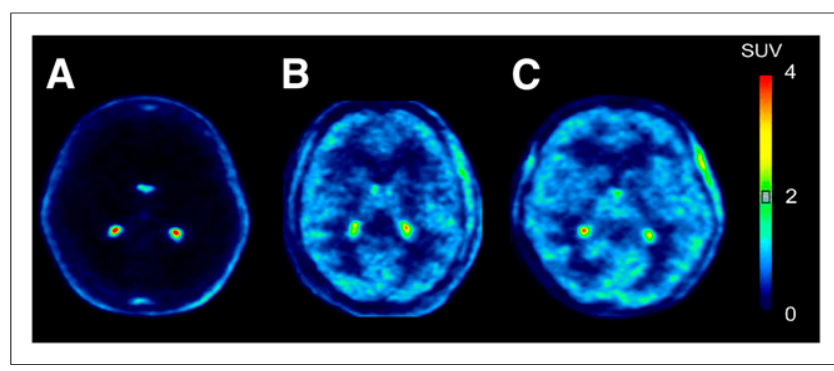

FIGURE 2. Representative images from ${ }^{11} \mathrm{C}$-dLop PET scans. Images are summed from 10 to $30 \mathrm{~min}$ after injection. Radioligand was injected either at baseline $(A)$ or during intravenous infusion of tariquidar at doses of 2 (B) and $4 \mathrm{mg} / \mathrm{kg}(\mathrm{C})$.

\section{DISCUSSION}

Performing ${ }^{11} \mathrm{C}$-dLop PET imaging during peak plasma concentrations of tariquidar resulted in greater P-gp inhibition at the BBB than injecting radiotracer after a delay. This concurrent administration strategy allowed use of a lower dose of tariquidar, expected to result in fewer adverse events. In contrast, neither $1,500 \mathrm{mg}$ of oral tariquidar nor oral disulfiram inhibited P-gp. These results suggest that the ability of tariquidar to inhibit P-gp at the BBB depends on the concentration of tariquidar in plasma. These results also suggest that oral tariquidar and disulfiram are not viable alternatives to intravenous tariquidar for inhibiting P-gp.

In a previous study from our laboratory, we injected ${ }^{11} \mathrm{C}$-dLop approximately $1 \mathrm{~h}$ after tariquidar infusion was completed, and other studies using ${ }^{11} \mathrm{C}$ - $(R)$-verapamil injected the radiotracer approximately $1-3 \mathrm{~h}$ after tariquidar $(7,8)$. One of the studies using ${ }^{11} \mathrm{C}-(R)$-verapamil showed that plasma concentrations of tariquidar declined rapidly after infusion and were followed by a long terminal phase, remaining stable at approximately $500 \mathrm{ng} / \mathrm{mL}$ (773 nM) for several hours (8). Therefore, in our prior study using ${ }^{11} \mathrm{C}$-dLop, we presumed that this terminal phase represented the concentration of tariquidar required to effectively inhibit P-gp at the BBB, given that plasma concentrations between 150 and 200 $\mathrm{ng} / \mathrm{mL}(230-310 \mathrm{nM})$ inhibit P-gp activity on leukocytes (9). However, subsequent studies demonstrated that greater concentrations of tariquidar are required to block P-gp activity at the BBB than on leukocytes $(14,15)$. Therefore, by administering ${ }^{11} \mathrm{C}$-dLop after completion of the tariquidar infusion, we may have missed the time window of maximal P-gp inhibition at the BBB. No previously published report or study has tested the efficacy of injecting substrate radiotracers during the tariquidar infusion, when plasma concentrations of this inhibitor peak $(\sim 1,000 \mathrm{ng} / \mathrm{mL}$ or $1,500 \mathrm{nM}$ during a $2 \mathrm{mg} / \mathrm{kg}$ infusion). In this study, ${ }^{11} \mathrm{C}$-dLop PET imaging was performed during infusion of 2 or $4 \mathrm{mg}$ of tariquidar per kilogram. We observed brain uptake comparable to that seen when performing ${ }^{11} \mathrm{C}$-dLop PET at $1 \mathrm{~h}$ after infusion of $6 \mathrm{mg}$ of tariquidar per kilogram (4). These results suggest that concurrent administration of radiotracer allows the use of lower doses of tariquidar while yielding a similar degree of P-gp inhibition. This method also reduces the amount of time required to administer tariquidar and obtain the ${ }^{11} \mathrm{C}$-dLop PET scan.

Studies have been conducted in drug-resistant cancer patients investigating the use of tariquidar to improve the efficacy of chemotherapy $(16,17)$. These studies have failed to increase penetration of chemotherapy drugs, perhaps because tariquidar was given at too low a dose $(<4 \mathrm{mg} / \mathrm{kg}$ intravenously) with too long an interval between administration of tariquidar and chemotherapy. The results from this study suggest that low-dose tariquidar has the potential to effectively inhibit P-gp and increase penetration of substrate medications when both the inhibitor and the active drug are simultaneously administered intravenously. Although it would be ideal to infuse tariquidar over the entire time course of the ${ }^{11} \mathrm{C}$ dLop PET scan, we infused at a fast rate over a short interval to achieve high plasma concentrations while avoiding toxicity. Peak uptake of ${ }^{11} \mathrm{C}$-dLop occurs within the first few minutes after injection with little washout thereafter $(6)$. Therefore, we don't expect the short tariquidar infusion time to have caused large variance in the $\mathrm{AUC}_{10-30}$ data. Rapid clearance of tariquidar from plasma may alter the kinetics of P-gp substrates that reversibly bind in the brain, however. Therefore, longer infusion time may be preferred for P-gp radiotracers such as ${ }^{11} \mathrm{C}-(R)$-verapamil.

The adverse events observed using high doses of tariquidar (4-6 $\mathrm{mg} / \mathrm{kg}$ intravenously) may have been caused by high amounts of the excipient, propylene glycol, delivered alongside tariquidar. In support of this theory, infrequent, mild side effects were observed when lower amounts of propylene glycol were delivered (intravenous tariquidar, $2 \mathrm{mg} / \mathrm{kg}$ ), and no side effects were observed when no propylene glycol was delivered at all $(1,500 \mathrm{mg}$ of oral tariquidar). However, oral tariquidar may be more tolerable simply because of its low bioavailability.

Oral tariquidar did not increase brain uptake of ${ }^{11} \mathrm{C}$-dLop, most likely because oral administration resulted in low plasma concentrations. Repeated administration of high oral doses of tariquidar could theoretically result in stable, high plasma concentrations over time. Whether this strategy would effectively inhibit P-gp and improve penetration of substrate medications in a clinical setting remains to be seen.

Similarly, disulfiram did not increase ${ }^{11} \mathrm{C}$-dLop brain uptake, even at a dose of $2.5 \mathrm{~g}$ over $4 \mathrm{~d}$. Disulfiram metabolites irreversibly inhibit P-gp in vitro (10), making this drug, which is inexpensive and commercially available, an attractive alternative to tariquidar for P-gp inhibition in a clinical setting. However, high doses of disulfiram have been associated with hepatic, neurologic, and dermatologic side effects (18). Our results suggest that either disulfiram metabolites do not inhibit P-gp at the BBB in vivo or doing so would require doses so large they would likely be toxic.

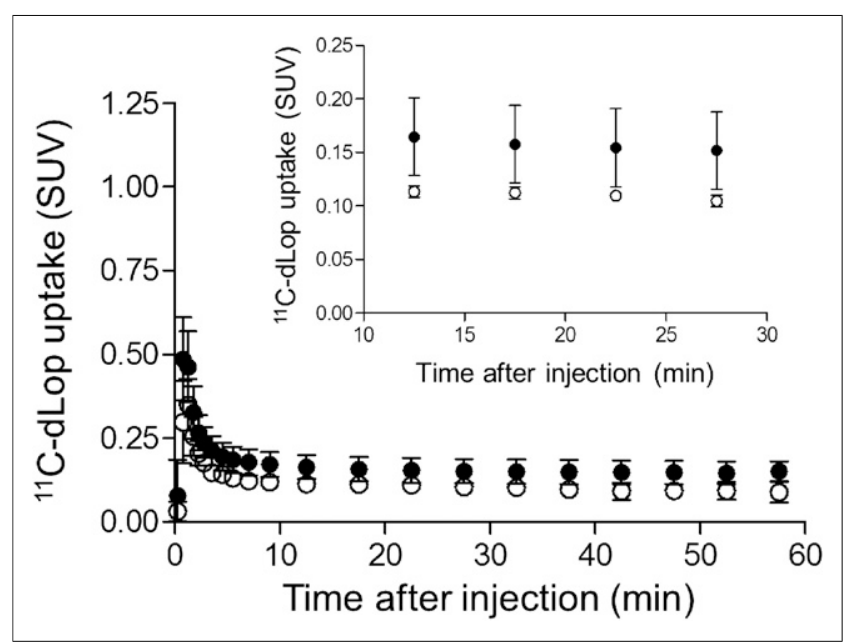

FIGURE 3. Composite neocortex time-activity curves showing brain uptake of radioactivity after ${ }^{11} \mathrm{C}$-dLop injection at baseline $(\mathbf{0})$ and after $1,500 \mathrm{mg}$ of oral tariquidar $(\bigcirc)$. Error bars denote SD. 


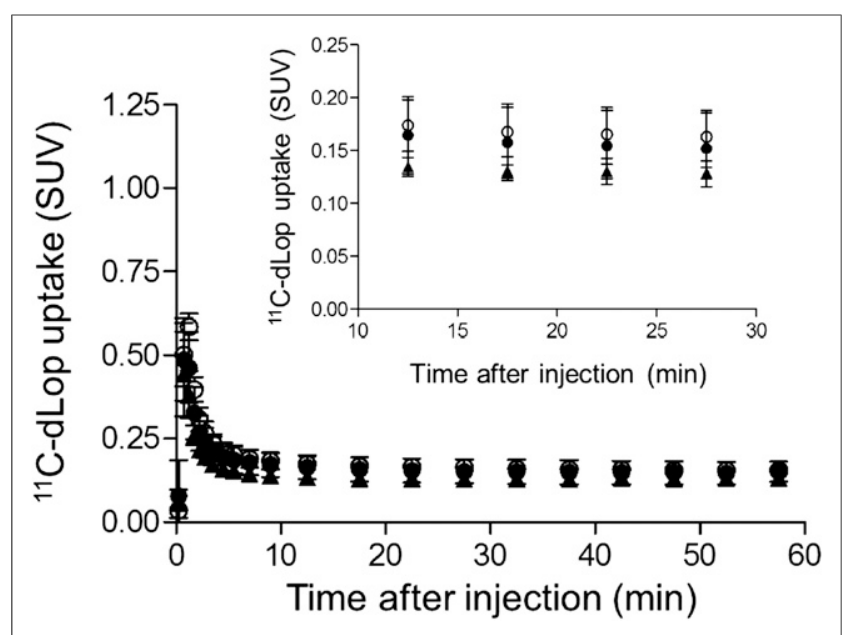

FIGURE 4. Composite neocortex time-activity curves showing brain uptake of radioactivity after injection of ${ }^{11} \mathrm{C}$-dLop at baseline $(\bullet)$ and after either 1-time dose of $500 \mathrm{mg}$ of disulfiram $(O)$ or $2.5 \mathrm{~g}$ of disulfiram over $4 \mathrm{~d}(\mathbf{\Lambda})$. Error bars denote SD.

We found that the tariquidar- ${ }^{11} \mathrm{C}$-dLop dose-response curve best fit a sigmoidal curve, suggesting that the PET response plateaued at high concentrations of tariquidar. Bauer et al. similarly showed that brain uptake of ${ }^{11} \mathrm{C}-(R)$-verapamil did not increase with doses of tariquidar greater than $6 \mathrm{mg} / \mathrm{kg}$ intravenously (7). Although these results suggest that plasma concentrations of tariquidar greater than $1,000 \mathrm{ng} / \mathrm{mL}$ completely inhibit P-gp at the $\mathrm{BBB}$, we do not know whether P-gp was maximally blocked in these experiments. We suspect that that the apparent plateau in ${ }^{11} \mathrm{C}$-dLop uptake was caused by noise and variability of the biologic response. In our earlier study, we estimated the first-pass extraction of ${ }^{11} \mathrm{C}$-dLop to be approximately $40 \%$ in monkey brain when the PET scan was performed after P-gp inhibition with (2R)anti-5-\{3-[4-(10,11-dichloromethanodibenzo-suber-5-yl)piperazin-1yl]-2-hydroxypropoxy \}quinoline trihydrochloride (5). Therefore, we would expect complete blockade of P-gp to result in high ${ }^{11} \mathrm{C}$ dLop uptake in the brain. Instead, the mean uptake was less than 1 SUV in the concurrent tariquidar group (Fig. 1), suggesting that P-gp was still preventing ${ }^{11} \mathrm{C}$-dLop from entering the brain to some extent at the highest concentrations of tariquidar achieved in this study.

Because both ${ }^{11} \mathrm{C}$-dLop and tariquidar are lysosomotropic (19), the apparent plateau in ${ }^{11} \mathrm{C}$-dLop uptake could have been due to tariquidar outcompeting ${ }^{11} \mathrm{C}$-dLop in lysosomes at higher concentrations of tariquidar. This possibility is speculative as we have no evidence that tariquidar enters the brain. Tariquidar is not only an inhibitor of P-gp but also a substrate for the breast cancer resistance protein $(20)$, which thereby blocks its entry into the brain. Further studies are required to determine whether tariquidar enters the brain at high plasma concentrations.

${ }^{11} \mathrm{C}$-dLop uptake in the pituitary did not increase after P-gp inhibition, suggesting that the lack of $\mathrm{BBB}$ around the pituitary allows entry of ${ }^{11} \mathrm{C}$-dLop independent of P-gp. That pituitary uptake was severalfold greater than brain uptake after tariquidar supports our conclusion that P-gp was not completely blocked in this study.

Although we didn't measure plasma radioactivity in most subjects in this study, we believe that increased brain radioactivity was due to P-gp inhibition, not increased ${ }^{11} \mathrm{C}$-dLop in plasma. We previously showed that intravenous tariquidar with delayed injection of radioligand increased brain radioactivity without increasing ${ }^{11} \mathrm{C}$-dLop in plasma (4). Also, we recently performed concurrent tariquidar- ${ }^{11} \mathrm{C}$-dLop PET in a separate study, and peak plasma concentration of ${ }^{11} \mathrm{C}$-dLop was similar (7.6 SUV) to reported values for subjects at baseline (4).

One limitation of this study is that we did not perform genetic testing to correct for the presence of known polymorphisms on the $A B C B 1$ gene. Some of these polymorphisms decrease P-gp expression or function (21) and may contribute to interindividual differences in PET response to tariquidar. Another limitation is that most PET scans were analyzed by calculating the $\mathrm{AUC}_{10-30}$ to avoid the need for arterial sampling. Full quantification of the PET data may have resulted in less variance in ${ }^{11} \mathrm{C}$-dLop uptake values. However, we do not think the simplified $\mathrm{AUC}_{10-30}$ measure introduces a large amount of error because our previous report showed a strong correlation between $\mathrm{AUC}_{10-30}$ and $K_{1}$ values for ${ }^{11} \mathrm{C}$-dLop (4). Moreover, because ${ }^{11} \mathrm{C}$-dLop rapidly clears from plasma (6), avoiding use of the arterial input function may actually reduce error in quantifying uptake of ${ }^{11} \mathrm{C}$-dLop in the brain. Another limitation is that free fraction of tariquidar was measured in only a subset of subjects. Because only tariquidar that is not bound to proteins in plasma is available to bind to and inhibit P-gp, only the free concentration of tariquidar would be expected to correlate with ${ }^{11} \mathrm{C}$-dLop uptake into the brain. However, we found little variance in the free fraction of tariquidar (coefficient of variation, $6 \%$ ), suggesting we would have seen a similar dose-response relationship using the free concentration of tariquidar.

\section{CONCLUSION}

Performing ${ }^{11} \mathrm{C}$-dLop PET imaging during peak plasma concentrations of tariquidar resulted in increased P-gp inhibition at the human BBB and allowed use of lower doses of the inhibitor. On the basis of prior studies in monkeys, we suspect that plasma concentrations of tariquidar did not fully block P-gp; however, higher doses of tariquidar would likely be associated with unacceptable side effects. This paradigm of concurrent administration of tariquidar and P-gp substrate drug may have potential for treating diseases associated with P-gp overexpression (e.g., multidrug resistant cancer).

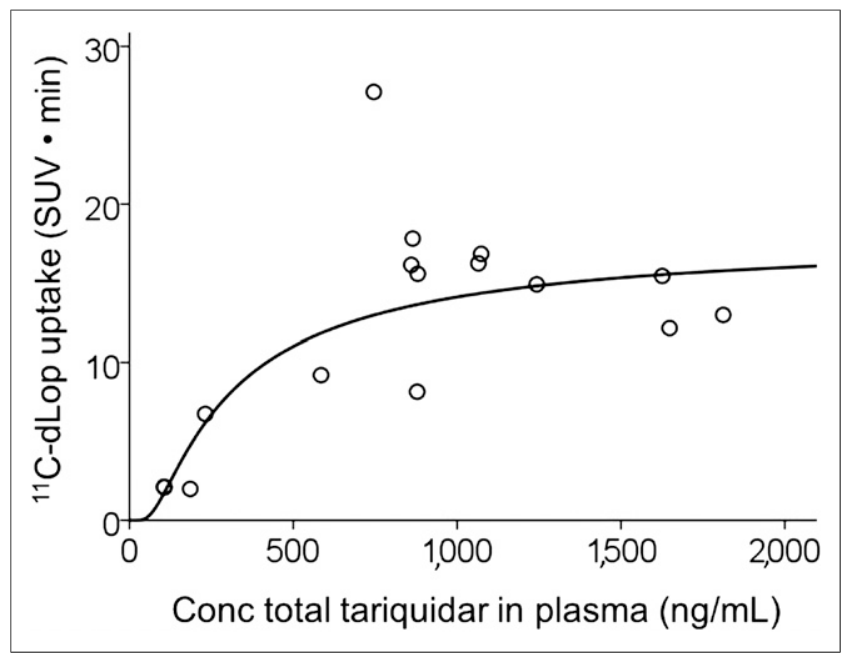

FIGURE 5. Dose-response curve for tariquidar and uptake of ${ }^{11} \mathrm{C}$ dLop in composite neocortex. Conc $=$ concentration. 


\section{DISCLOSURE}

The costs of publication of this article were defrayed in part by the payment of page charges. Therefore, and solely to indicate this fact, this article is hereby marked "advertisement" in accordance with 18 USC section 1734. This work was supported by the Intramural Research Program of the National Institute of Mental Health, National Institutes of Health (IRP-NIMH-NIH) under clinicaltrials.gov identifier NCT00605254 (protocol 08-M-0062). No other potential conflict of interest relevant to this article was reported.

\section{ACKNOWLEDGMENTS}

We thank AzaTrius Pharmaceuticals for supplying tariquidar; Judith Starling, George Grimes, and Gerald Overman for formulating tariquidar; Jinsoo Hong and Yi Zhang for assistance in the production of radiotracer; David Luckenbaugh and Seonjoo Lee for assistance with statistical analysis; Ioline Henter for excellent editorial assistance; and Maria D. Ferraris-Araneta, Denise RallisFrutos, Yulin Chu, Barbara Scepura, Emily Page, Gerald Hodges, Robert Gladding, and the NIH PET Department for successfully completing the PET studies. The internal standard used in the liquid chromatography-tandem mass spectrometry method was synthesized by Sanjay Telu.

\section{REFERENCES}

1. Gottesman MM. Mechanisms of cancer drug resistance. Annu Rev Med. 2002;53: 615-627.

2. Kalvass JC, Pollack GM. Kinetic considerations for the quantitative assessment of efflux activity and inhibition: implications for understanding and predicting the effects of efflux inhibition. Pharm Res. 2007;24:265-276.

3. Kannan P, John C, Zoghbi SS, et al. Imaging the function of P-glycoprotein with radiotracers: pharmacokinetics and in vivo applications. Clin Pharmacol Ther. 2009;86:368-377.

4. Kreisl WC, Liow JS, Kimura N, et al. P-glycoprotein function at the blood-brain barrier in humans can be quantified with the substrate radiotracer ${ }^{11} \mathrm{C}-\mathrm{N}$-desmethylloperamide. J Nucl Med. 2010;51:559-566.

5. Liow JS, Kreisl W, Zoghbi SS, et al. P-glycoprotein function at the blood-brain barrier imaged using ${ }^{11} \mathrm{C}-\mathrm{N}$-desmethyl-loperamide in monkeys. J Nucl Med. 2009;50:108-115.
6. Seneca N, Zoghbi SS, Liow JS, et al. Human brain imaging and radiation dosimetry of ${ }^{11} \mathrm{C}-\mathrm{N}$-desmethyl-loperamide, a PET radiotracer to measure the function of P-glycoprotein. J Nucl Med. 2009;50:807-813.

7. Bauer M, Zeitlinger M, Karch R, et al. Pgp-mediated interaction between (R)$\left[{ }^{11} \mathrm{C}\right]$ verapamil and tariquidar at the human blood-brain barrier: a comparison with rat data. Clin Pharmacol Ther. 2012;91:227-233.

8. Wagner CC, Bauer M, Karch R, et al. A pilot study to assess the efficacy of tariquidar to inhibit P-glycoprotein at the human blood-brain barrier with (R)- ${ }^{11}$ C-verapamil and PET. J Nucl Med. 2009;50:1954-1961.

9. Stewart A, Steiner J, Mellows G, Laguda B, Norris D, Bevan P. Phase I trial of XR9576 in healthy volunteers demonstrates modulation of P-glycoprotein in CD56+ lymphocytes after oral and intravenous administration. Clin Cancer Res. 2000;6:4186-4191.

10. Loo TW, Bartlett MC, Clarke DM. Disulfiram metabolites permanently inactivate the human multidrug resistance P-glycoprotein. Mol Pharm. 2004;1:426-433.

11. Loo TW, Clarke DM. Blockage of drug resistance in vitro by disulfiram, a drug used to treat alcoholism. J Natl Cancer Inst. 2000;92:898-902.

12. Sauna ZE, Peng XH, Nandigama K, Tekle S, Ambudkar SV. The molecular basis of the action of disulfiram as a modulator of the multidrug resistance-linked ATP binding cassette transporters MDR1 (ABCB1) and MRP1 (ABCC1). Mol Pharmacol. 2004;65:675-684.

13. Lazarova N, Zoghbi SS, Hong J, et al. Synthesis and evaluation of [N-methyl- $\left.{ }^{11} \mathrm{C}\right]$ $\mathrm{N}$-desmethyl-loperamide as a new and improved PET radiotracer for imaging P-gp function. J Med Chem. 2008;51:6034-6043.

14. Choo EF, Kurnik D, Muszkat M, et al. Differential in vivo sensitivity to inhibition of P-glycoprotein located in lymphocytes, testes, and the blood-brain barrier. J Pharmacol Exp Ther. 2006;317:1012-1018.

15. Kurnik D, Sofowora GG, Donahue JP, et al. Tariquidar, a selective P-glycoprotein inhibitor, does not potentiate loperamide's opioid brain effects in humans despite full inhibition of lymphocyte P-glycoprotein. Anesthesiology. 2008;109:1092-1099.

16. Pusztai L, Wagner P, Ibrahim N, et al. Phase II study of tariquidar, a selective P-glycoprotein inhibitor, in patients with chemotherapy-resistant, advanced breast carcinoma. Cancer. 2005;104:682-691.

17. Abraham J, Edgerly M, Wilson R, et al. A phase I study of the P-glycoprotein antagonist tariquidar in combination with vinorelbine. Clin Cancer Res. 2009; 15:3574-3582.

18. Enghusen Poulsen H, Loft S, Andersen JR, Andersen M. Disulfiram therapy: adverse drug reactions and interactions. Acta Psychiatr Scand Suppl. 1992;369:59-65.

19. Kannan P, Brimacombe KR, Kreisl WC, et al. Lysosomal trapping of a radiolabeled substrate of P-glycoprotein as a mechanism for signal amplification in PET. Proc Natl Acad Sci USA. 2011;108:2593-2598.

20. Kannan P, Telu S, Shukla S, et al. The "specific" P-glycoprotein inhibitor tariquidar is also a substrate and an inhibitor for breast cancer resistance protein (BCRP/ABCG2). ACS Chem Neurosci. 2011;2:82-89.

21. Fromm MF. The influence of MDR1 polymorphisms on P-glycoprotein expression and function in humans. Adv Drug Deliv Rev. 2002;54:1295-1310. 